\title{
Factors affecting proprioceptive adaptation to prismatic displacement
}

\author{
BENJAMIN WALLACE \\ Cleveland State University, Cleveland, Ohio 44115
}

\begin{abstract}
Two prism displacement experiments were conducted to determine the effects of reducing proprioceptive feedback on resultant adaptation magnitude. In Experiment 1, proprioceptive reduction was produced by requesting subjects to employ passive (vs. active) and/or fast- (vs. slow-) paced arm movement during prism exposure. When both of these conditions were present, a significant reduction in the magnitude of proprioceptive adaptation and a significant increase in the magnitude of visual adaptation were produced. In Experiment 2, hypnotic anesthesia was employed to reduce felt sensation in an adapting limb during a prism displacement situation. This manipulation reduced proprioceptive adaptation to a nonsignificant level. The combined results of the two experiments reveal several conditions that can serve to reduce proprioceptive adaptation during prism displacement.
\end{abstract}

Despite a series of early experiments by Held and his collaborators (Held \& Freedman, 1963; Held \& Gottlieb, 1958; Held \& Hein, 1958; Held \& Schlank, 1959 ) indicating that adaptation to prismatic displacement requires actively controlled and observed arm movement, it is now accepted that adaptation can occur with passive arm movement under certain conditions (Baily, 1972; Fishkin, 1969; Foley \& Maynes, 1969; Melamed, Halay, \& Gildow, 1973; Weinstein, Sersen, \& Weinstein, 1964; Welch, 1978). These conditions generally require that a subject be able to localize a visual target position during passive movement (Melamed et al., 1973; Pick \& Hay, 1965) and that such movements be accomplished under conditions of terminal display in which arm movement can be observed only at the final stage of a localization action (Baily, 1972). When these conditions are present, information about the discordance between vision and proprioception is available and the subject can make use of this information to adapt to the prism displacement.

Baily (1972) also found that speed of arm movement in a localization task has an effect on resultant adaptation to prism displacement. During prism exposure, adaptation was less when a subject performed a fast, reaching movement toward a target location than when he performed a slow, reaching action. Baily interpreted this as indicating that fast movements are basically automatic and do not require proprioceptive recalibration for adaptation to be produced. However, Baily assessed only eye-hand

This research was supported by Grant MH-34383-01 from the National Institute of Mental Health. The assistance of Diane Sanders in collecting the data is gratefully acknowledged. Reprint requests should be sent to the author, Department of Psychology, Cleveland State University, Cleveland, Ohio 44115. coordination, which is measured by requesting a subject to localize a visual target before and after an exposure to prism displacement. The measure is a difference score between preexposure and postexposure localization of a target position and is typically such that postexposure reaching is in the direction opposite to the prism base displacement. For this reason, this measure is referred to as the negative aftereffect (NA). This measure has been found to be multidetermined, or the result of several inputs (Redding \& Wallace, 1976, 1978; Wallace \& Fisher, 1979; Wallace \& Garrett, 1975; Wallace \& Redding, 1979; Wilkinson, 1971). As a result, although Baily found that adaptation to prismatic displacement could be produced with passive arm movement under the conditions previously specified, it is unclear to what extent proprioception and vision (the two components comprising the NA) played roles in the total adaptation process. Specifically, although Baily reported that slow arm movement during prism exposure produced a greater NA than did fast arm movement, it is not clear to what extent this finding was the result of proprioceptive input or of visual input.

To clarify the results reported by Baily, Experiment 1 assessed magnitude of adaptation to prismatic displacement as a function of arm-movement specificity. Two conditions of arm movement were considered in terms of their roles in the adaptation process. These included (1) arm-movement control, either active or passive, and (2) arm-movement pace, either slow or fast. By manipulating these arm-movement conditions, it may be possible to specify more accurately the conditions under which adaptation to prismatic displacement occurs and to what extent. It should also be possible to determine the magnitude of adaptation for the various components specified. These components include the NA, the measure of 
eye-hand coordination, and the two components that theoretically comprise this value. These are referred to as the proprioceptive shift (PS) and the visual shift (VS). The PS is assessed as the difference between preexposure and postexposure localization of the straight-ahead position in space. The VS is a value determined by the difference in preexposure and postexposure target alignment performance. Since NA is multidetermined (NA = PS + VS), Experiment 1 also assessed the relative contribution of the various armmovement conditions to the adaptation process.

\section{EXPERIMENT 1}

\section{Method}

Subjects. Forty-eight right-handed undergraduate volunteers from introductory psychology sections served as subjects. All individuals had normal motor dexterity and normal, uncorrected visual acuity.

Apparatus. The apparatus was similar to one employed by Wallace and Fisher (1979). Basically, this consisted of a threelayer, rectangular, wooden, box-like frame $(77 \mathrm{~cm}$ high, $62 \mathrm{~cm}$ wide, and $62 \mathrm{~cm}$ deep), open on the side facing the subject. When subjects placed one arm within the structure, on the lowest layer, the arm was not visible. In this area, during test situations, arm position could be noted by the experimenter by determining its location along a 180-deg arc, calibrated in 1-deg increments. During exposure, placement of the subject's arm upon the center layer permitted the individual to perceive arm movement only at the terminus of a response action. Observation of arm movement throughout most of the exposure session was prevented by a third layer, located $12 \mathrm{~cm}$ directly above the second layer of the apparatus. When a subject was to localize a visible target $(.2 \times 8 \mathrm{~cm})$, this stimulus was mounted symmetrically with respect to body position on a backboard attached to the top layer of the apparatus. Attached to this apparatus was a Marietta Instruments combination head- and chinrest.

Procedure. The procedure was the same as that employed by Wallace (1978). Specifically, each subject was tested individually by being led to the experimental room and seated in a chair before the apparatus. Risley prisms set to 0 diopter and mounted in welder's goggles were then placed over the subject's eyes. The subject's head was then positioned in the head-and chinrest, and each individual was asked to perform each of three localization tests.

The PS test required the subject to place his dominant arm on the lower layer of the apparatus and to point to the position in space that he believed to be straight ahead of his nose. This task was accomplished 10 times with vision occluded (a blindfold was placed over the goggles).

The NA test was similar, except the subject was not blindfolded. In this test, the subject pointed to a visible, vertical target $(.2 \times 8 \mathrm{~cm})$ located in the physical straight-ahead position. However, pointing accuracy was not known to the subject, since his hand was on the lower layer of the apparatus. Ten measures were taken for this localization task.

The VS test required no arm movement. Instead, the experimenter introduced a moving, visible, vertical target $(.2 \times 8 \mathrm{~cm})$ on the top layer of the apparatus. When the experimenter moved this target in a lateral fashion across the subject's visual field, the subject simply indicated when the target appeared to be straight ahead of his nose. A total of 10 trials were given on this test. Order of starting position of target movement was random across the 10 trials. Also, randomization was applied to the 30 combinations of 10 trials and the three kinds of tests for the 48 subjects.

After establishing the three baseline measures, the subject was instructed to place his arm between the second and third layers of the apparatus. The Risley prisms were then adjusted to 20-diopter, base-right, while the subject had his eyes closed. He was then instructed to open his eyes and to begin pointing (active movement), or to allow the experimenter to control his pointing (passive movement), to a target $(.2 \times 8 \mathrm{~cm})$ located in the physical straightahead position. Passive movement was accomplished by first requesting the subject to make his arm as limp as possible. The experimenter then held the subject's arm and physically moved it. A pointing finger generally ended up near or on the target location on each point. ${ }^{1}$ Such pointing was performed in a ballistic fashion for $15 \mathrm{~min}$. Error-corrective feedback was provided only at the terminus of a prism exposure movement toward the target location. The rate of movement was regulated by a metronome set so that one movement cycle ("back and forth" action) occurred in each 6-sec period (slow movement) or in each 2 -sec period (fast movement). ${ }^{2}$

Following the prism-exposure task, the subject was instructed to close his eyes, and the experimenter set the subject's prisms to 0 diopter as in the preexposure phase. The subject then opened his eyes and performed the three preexposure localization tasks 10 times each.

\section{Results and Discussion}

A preliminary analysis of the data indicated the absence of a significant trials effect for each of the three coordination tasks. As a result, subsequent analyses were restricted to the averaged difference scores between preexposure and postexposure in pointing accuracy to the target position (NA), in pointing accuracy to the straight-ahead position (PS), and in target alignment to the straight-ahead position (VS). An adaptive shift for the target-pointing response and for the straight-ahead pointing response required postexposure pointing to be in the direction opposite to the prism displacement; an adaptive shift for target alignment required postexposure responding to be in the same direction as the prism base orientation (Harris, 1965; Welch, 1978).

An analysis of variance performed on the averaged difference scores between preexposure and postexposure pointing and/or responding produced a significant three-way interaction [Arm Movement Control by Arm Movement Pace by Adaptation Type (NA, PS, and VS), $F(2,88)=5.43, p<.006$ ]. Table 1 summarizes the mean values of adaptation (NA, PS, and VS) within this interaction. As expected, the greatest NA and PS magnitudes were produced when active, slow arm movement was employed during prism exposure (Newman-Keuls, $\mathrm{p}<.001$ ). This finding confirms the results of early studies reported by Held and his colleagues with respect to the superiority of active movement over passive movement in the production of NA and PS in a prism adaptation task. Also, for this condition, the additive model of prism adaptation (Wilkinson, 1971) was confirmed. Specifically, NA = PS + VS.

The additive model also held for the passive, slowmovement condition. Furthermore, the prediction made by Baily (1972) was also confirmed, since passive, slow movement produced a significant level of both NA and PS adaptation, although each was sig- 
Table 1

Mean Adaptation Magnitude as a Function of Arm Movement Control (AMC) and Arm Movement Pace (AMP)

\begin{tabular}{|c|c|c|c|c|c|c|c|}
\hline \multirow[b]{3}{*}{ AMP } & \multicolumn{6}{|c|}{ Adaptation Type } & \multirow[b]{3}{*}{ A } \\
\hline & \multicolumn{2}{|c|}{ NA } & \multicolumn{2}{|c|}{ PS } & \multicolumn{2}{|c|}{ VS } & \\
\hline & Mean & SD & Mean & SD & Mean & SD & \\
\hline & \multicolumn{7}{|c|}{ Active AMC } \\
\hline Slow & $4.07^{*}$ & 1.35 & $3.03 *$ & 1.31 & $1.14^{*}$ & .64 & + \\
\hline \multirow[t]{2}{*}{ Fast } & $2.15^{*}$ & .97 & .63 & .59 & $1.71^{*}$ & .63 & + \\
\hline & \multicolumn{7}{|c|}{ Passive AMC } \\
\hline Slow & $2.47^{*}$ & .73 & $1.19^{*}$ & .43 & $1.30^{*}$ & .52 & + \\
\hline Fast & .83 & .80 & .57 & .39 & $2.41^{*}$ & 1.39 & - \\
\hline
\end{tabular}

Note-A = additivity. The $N A$ value did not significantly differ from the combined $P S+V S$ value if additivity was present (+indicates presence; - indicates absence). $\quad{ }^{*} p<.05$ or better; analyses were made by two-tailed t tests between preexposure and postexposure pointing scores $(d f=11)$.

nificantly reduced (Newman-Keuls, $\mathrm{p}<.001$ ) in magnitude when compared with the respective measures as established during the active, slow-movement condition. The magnitude of VS in the active, slowmovement condition and in the passive, slow-movement condition did not differ significantly. Thus, the substitution of passive movement for active movement in a slow-paced coordination task appears to have directly affected the magnitude of the PS component.

When the ballistic pointing pace was accelerated, this also served to reduce the magnitude of produced PS (Newman-Keuls, $p<.001$ ). In fact, for both active and passive movement, the magnitude was reduced to a nonsignificant level, while the VS magnitude was significantly increased (Newman-Keuls, $p<.01$ ). For the active, fast-movement condition, PS was significantly reduced (Newman-Keuls, $\mathrm{p}<.01$ ) and VS was significantly increased (Newman-Keuls, $\mathrm{p}<.01$ ) as compared with the measures produced in the active, slow-movement condition, respectively. This was also the case when the PS and VS values in the passive, fast-movement condition were compared with those in the passive, slow-movement condition. This increase in VS when PS is decreased replicates a finding reported by Wallace (1975).

For the active, fast-movement condition, the additive model held, with the value of NA being determined primarily by VS. However, this was not the case for the passive, fast-movement condition, although PS was decreased in magnitude to a nonsignificant level, while VS was enhanced in magnitude. The reason for this nonadditivity may relate to the possibility that during fast, passive-exposure arm movement, a subject may not have worked at the eye-hand coordination test or was confused in performing it. If this is so, one might expect a decreased level of NA, since the subject would not adapt to target pointing activity. Such confusion might then produce nonadditivity of the adaptation com- ponents. However, subject confusion should also produce increased variability associated with NA adaptation. This was not found. Therefore, the reasons for the nonsignificant level of NA and resultant nonadditivity are unclear.

It appears, then, that when the two conditions (passive and fast arm movement) that have been found to reduce proprioceptive adaptation are simultaneously present in an adaptation experiment, adaptation is primarily of the VS variety. This finding lends credence to Baily's theory that fast-paced movement is automatic and does not require proprioceptive recalibration. This results in a nonsignificant level of PS being produced, both for active and for passive movement.

If, as Baily predicted, proprioceptive adaptation is greatest in magnitude when arm-movement control is both active and slowly paced, in addition to reducing magnitude of such adaptation by requiring passive and/or fast arm movement, another procedure for reducing proprioceptive feedback would be to introduce hypnotic anesthesia in the adapting arm. Several studies (Wallace \& Fisher, 1979; Wallace \& Garrett, 1973; Welch \& Snyder, cited in Welch, 1978 , p. 27) had previously shown that the induction of hypnotic anesthesia in an adapting limb reduces, to a nonsignificant level, the subsequent magnitude of proprioceptive adaptation as measured from that limb. If proprioception was the major factor producing the difference between active and passive arm movement and between slow and fast arm movement in the Baily study, hypnotic anesthesia could be employed to help verify that conclusion. Also, if PS was reduced and NA remained constant, VS should be increased, as predicted by the additive model. These possibilities were considered in Experiment 2.

\section{EXPERIMENT 2}

\section{Method}

Subjects. Thirty-six volunteers from introductory psychology classes served as subjects. Prior to experimental participation, each subject had participated in a mass-testing session in which the Harvard Group Scale of Hypnotic Susceptibility: Form A (Shor \& Orne, 1962) was administered. Twenty-four subjects scored high (9-12) on the test, while 12 subjects scored low (0-3).

Apparatus. The apparatus was identical to the one employed in Experiment 1.

Procedure. Each subject was tested individually by being led to an experimental room and seated in a chair before the apparatus. The subject's head was then positioned in the head- and chinrest. One of two experimenters (Experimenter 1) then placed Risley rotating prisms, set to 0 diopter and mounted in welder's goggles, over the subject's eyes. After this procedure, each subject was required to perform the same three localization tasks as in Experiment 1 to determine preexposure baselines for target-pointing, straight-ahead-pointing, and target-alignment accuracy. Order of preexposure task performance was random.

Following the establishment of the NA, PS, and VS baselines, the Risley prisms were removed from the subject's eyes and were replaced with a blindfold. The subjects were then moved to a different room. At this point, if the subjects were judged to be 
high in hypnotic susceptibility by virtue of their hypnosis test scores, they either had their dominant arm hypnotically anesthetized or were requested to role-play the sensation of having their dominant limb so anesthetized. Twelve subjects participated in each of these two conditions. The subjects were sufficiently informed with respect to the consequences of induced, hypnotic anesthesia. They were told that the arm would feel totally numb and absent of all felt sensations. The high-susceptibility, role-playing group served as a control to be certain that hypnotic susceptibility, per se, was not an influencing factor in the experiment. The subjects who scored low on the attribute of hypnotic susceptibility were requested to role-play the sensation of having their dominant arms hypnotically anesthetized. Twelve subjects were assigned to this condition, one that is traditionally employed when hypnosis is used as a manipulation. Success of the hypnotic anesthesia manipulation was determined by requesting the subject to point to his nose (the blindfold prevented the subject from observing this action). The ability to perform this simple, proprioceptive task has been shown to be affected by the implementation of hypnotic anesthesia in the pointing limb. When this manipulation is in effect, subjects either miss the nose location or exhibit a prolonged reaction time from movement initiation to nose localization (Wallace \& Hoyenga, 1980). This manipulation check was conducted by the second experimenter (Experimenter 2) on all subjects regardless of condition assignment. Only hypnotically anesthetized subjects responded in error to the nose-touch task. Experimenter 1 was blind with respect to subject group assignment and with respect to the anesthesia manipulation check.

Following anesthesia induction (real or role-played), the subject was returned to the original experimental room which contained the previously described apparatus. Upon return, the subject was again seated in the chair before the apparatus. At this point, Experimenter 1 asked the subject to close his eyes. The blindfold was then removed and replaced with prisms, set to 20 diopter, base right. The subject was then requested to open his eyes and to point to the target as described in Experiment $1 .^{3}$ Pointing was performed in a ballistic fashion such that the subject extended his arm away from his body and then brought it back. This backand-forth action was performed at the slow pace described in Experiment 1. During the exposure, terminal pointing was performed when the consequences of a point were perceived only at the terminus of the action. The subject was requested to perform this task for 15 min.

After the exposure period, the prisms were removed from the subject's eyes and replaced with a blindfold. The subject was requested to keep his eyes closed during this operation. He was then led to the experimental room where anesthesia (real or roleplayed) had originally been induced. Experimenter 2 then removed the anesthesia from those who actually received this manipulation or informed role-playing subjects that they no longer needed to pretend that their dominant arms were hypnotically anesthetized. Following the removal of the anesthesia, which required approximately $30 \mathrm{sec}$ for completion, Experimenter 2 asked the subjects to again perform the nose-touch task. At this point in the experiment, all subjects performed the task veridically.

Immediately after anesthesia removal, the blindfolded subject was returned to the experimental room containing the apparatus. Upon arrival, Experimenter 1 removed the subject's blindfold and replaced it with prisms set to 0 diopter. Each individual was then requested to perform the preexposure localization tasks, five times each, in a postexposure session. Level of adaptation was specified as the signed difference between mean preexposure and mean postexposure localization measures.

\section{Results and Discussion}

As in Experiment 1, a preliminary analysis of the data failed to produce a significant trials effect for any of the three localization tasks. As a result, sub-
Table 2

Mean Adaptation Magnitude as a Function of Induced

Hypnotic Anesthesia in an Adapting Limb During Active, Slow-Paced Arm Movement

\begin{tabular}{|c|c|c|c|c|c|c|c|c|}
\hline \multirow[b]{3}{*}{$\mathrm{HL}$} & \multirow[b]{3}{*}{ HA } & \multicolumn{6}{|c|}{ Adaptation Type } & \multirow[b]{3}{*}{ A } \\
\hline & & \multicolumn{2}{|c|}{ NA } & \multicolumn{2}{|c|}{ PS } & \multicolumn{2}{|c|}{ VS } & \\
\hline & & Mean & $\mathrm{SD}$ & Mean & SD & Mean & $\mathrm{SD}$ & \\
\hline High & Actual & $3.18^{*}$ & 1.71 & .30 & .30 & $2.88^{*}$ & 1.45 & + \\
\hline High & Role-Played & $3.43^{*}$ & 1.91 & $2.28^{*}$ & 1.90 & $1.17^{*}$ & 1.32 & + \\
\hline Low & Role-Played & $3.62 *$ & 1.33 & $2.63^{*}$ & 1.89 & $1.01^{*}$ & 1.33 & + \\
\hline
\end{tabular}

Note-HL $=$ hypnotic susceptibility level; HA = hypnotic anesthesia type; $A=$ additivity. The NA value did not significantly differ from the combined $P S+V S$ value if additivity was present (indicated by + ). $\quad * p<.05$ or better; analyses were made by two-tailed t tests between preexposure and postexposure pointing scores $(d f=11)$.

sequent analyses were restricted to the averaged difference scores between preexposure and postexposure in pointing accuracy to the target position (NA), in pointing accuracy to the straight-ahead position (PS), and in target alignment to the straight-ahead position (VS).

Dependent-measure $t$ tests were applied to the difference between preexposure and postexposure scores (shifts). In a comparison of the NA, PS, and VS values (see Table 2) for the adapting limb, each group demonstrated a significant NA. The NA values in the three respective groups did not significantly differ from each other. Significant PS values were also produced for the two groups of subjects who roleplayed hypnotic anesthesia in their adapting limbs, and these values did not significantly differ from each other. The PS value for the group of individuals who received actual hypnotic anesthesia was not significant. This absence of a significant PS replicates a number of previous studies (Wallace \& Fisher, 1979; Wallace \& Garrett, 1973, 1975; Welch \& Snyder, cited in Welch, 1978, p. 27). In addition, when PS is reduced to a nonsignificant level, there is an accompanying increase in the magnitude of VS. Only in the group of subjects who received actual hypnotic anesthesia did the magnitude of VS exceed the magnitude of PS. In all three groups of subjects, the additive model (Wallace, 1977; Wilkinson, 1971) of prism adaptation held.

\section{CONCLUSIONS}

Experiment 1 demonstrated that two conditions appear to reduce the magnitude of PS when they are present in a prism-adaptation experiment. These are (1) requiring a subject to perform a localization action with a passively controlled limb and (2) performing such a localization action at a fast pace. When either or both of these conditions prevailed, the magnitude of PS was either significantly reduced (as in the 
passive, slow-pace condition) or was nonsignificant (as in both fast-pace conditions). The magnitude of VS also appeared to increase when PS was decreased.

In Experiment 2, the reduction of felt sensation in an adapting limb by means of hypnotic anesthesia also reduced PS to a nonsignificant level while increasing the magnitude of VS. As a result, PS appears to be produced by at least three inputs: (1) the ability to feel sensations in the adapting limb, (2) the ability to be in control of movement of that limb, and (3) the ability to attend to such movement, which appears to be optimized when such movement is slowly paced. When one or more of these inputs is reduced, eliminated, or changed during a period of adapting to a rearranged environment, PS is reduced in magnitude. In addition, this reduction appears to be accompanied by an increase in the magnitude of VS. This indicates that when proprioceptive sources of information are reduced or cannot be employed in adapting to a prism distortion, available visual information appears to play a role in permitting adaptation to occur. Furthermore, unless confusion is of a high magnitude, as was likely the case in the passive, fast-pace condition in Experiment 1, the additive model of prismatic adaptation appears to hold. Therefore, this model appears to be a useful tool for predicting magnitude of PS and VS when proprioceptive sources of input are not present or are made difficult to employ in adaptation.

\section{REFERENCES}

BAlly, J. S. Adaptation to prisms: Do proprioceptive changes mediate adapted behavior with ballistic arm movements? Quarterly Journal of Experimental Psychology, 1972, 24, 8-20.

Fisikin, S. M. Passive vs. active exposure and other variables related to the occurrence of hand adaptation to lateral displacement. Perceptual and Motor Skills, 1969, 29, 291-297.

FolEY, J. E., \& MAYNES, F. J. Comparison of training methods in the production of prism adaptation. Journal of Experimental Psychology, 1969, 81, 151-155.

Harris, C. S. Perceptual adaptation to inverted, reversed, and displaced vision. Psychological Review, 1965, 72, 419-444.

Held, R., \& Freedman, S. J. Plasticity in human sensorimotor control. Science, 1963, 142, 455-462.

Held, R., \& GottliteB, N. Technique for studying adaptation to disarranged hand-eye coordination. Perceptual and Motor Skills, $1958,8,83-86$.

Held, R., \& Hein, A. Adaptation to disarranged hand-eye coordination contingent upon reafferent stimulation. Perceptual and Motor Skills, 1958, 8, 87-90.

Held, R., \& Schlank, M. Adaptation to disarranged eye-hand coordination in the distance dimension. American Journal of Psychology, 1959, 72, 603-605.

Mei.amed, L. E., Halay, M., \& Gildow, J. W. Effect of external target presence on visual adaptation with active and passive movement. Journal of Experimental Psychology, 1973, 98, 125-130.

Pick, H. L., Jr., \& HaY, J. C. A passive test of the Held reafference hypothesis. Perceptual and Motor Skills, 1965, 20, 1070-1072.

Rendinc, G. M., \& Wal.i,Ace, B. Components of displacement adaptation in acquisition and decay as a function of hand and hall exposure. Perception \& Psychophysics, 1976, 20, 453-459.
Rendinc, G. M., \& Wallace, B. Sources of overadditivity in prism adaptation. Perception \& Psychophysics, 1978, 24, 58-62.

Shor, R. E., \& Orne, E. C. Harvard Group Scale of Hypnotic Susceptibility: Form A. Palo Alto, Calif: Consulting Psychologists Press, 1962.

WALLACE, B. Preexposure pointing frequency effects on adaptation to prismatic viewing. Perception \& Psychophysics, 1974, 15, 26-30.

Wallace, B. Prism adaptation to moving and stationary target exposures. Perception, 1975, 4, 341-347.

Wallace, B. Stability of Wilkinson's linear model of prism adaptation over time for various targets. Perception, 1977, 6, 145-151.

WALLACE, B. Visuomotor coordination and intermanual transfer for a proprioceptive reaching task. Journal of Motor Behavior, $1978,10,139-147$.

Wallace, B., \& Fisher, L. E. Proprioception and the production of adaptation and intermanual transfer 10 prismatic displacement. Perception \& Psychophysics, 1979, 26, 113-117.

Wallace, B., \& Garrf,Tt, J. B. Reduced felt arm sensation effects on visual adaptation. Perception \& Psychophysics, 1973, 14, 597-600.

Wallace, B., \& Garkett, J. B. Perceptual adaptation with selective reductions of felt sensation. Perception, 1975, 4, 437-445.

Wallace, B., \& Hoytinga, K. B. Production of proprioceptive errors with induced hypnotic anesthesia. International Journal of Clinical and Experimental Hypnosis, 1980, 28, 140-147.

Wallace, B., \& Rendinci, G. M. Additivity in prism adaptation as manifested in intermanual and interocular transfer. Perception \& Psychophysics, 1979, 25, 133-136.

Weinstein, S., Sersen, E. A., \& Wrinstein, D. S. An attempt to replicate a study of disarranged eye-hand coordination. Perceptual and Motor Skills, 1964, 18, 629-632.

We LCh, R. B. Perceptual adaptation: Adapting to altered sensory environments. New York: Academic Press, 1978.

Wilkinson, D. A. Visual-motor control loop: A linear system? Journal of Experimental Psychology, 1971, 89, 250-257.

\section{NOTES}

1. Pointing errors during prism exposure were not recorded. However, initial points were typically in the same direction as prism displacement (to the left of target position). This was evident for both actively controlled movement conditions but was most pronounced for the fast-paced situation. This was most likely the result of slow-paced movements' being corrected while in motion toward the target position. Fast-paced movements could obviously not be so easily corrected. With practice, errors tended to disappear for both active-movement groups. It was not possible to control pointing-error type and magnitude for the passivemovement subjects.

2. The speed with which the pointing movement was completed may appear to be confounded with the number of pointing responses during the exposure period. However, frequency of pointing actions has been shown to increase, rather than decrease, adaptation magnitude of NA and only when applied to nonmedianplane targets (Wallace, 1974). Therefore, frequency of exposure pointing would not appear to be an important factor in contributing to the effect reported here.

3. Exposure pointing errors that involved missing the target location appeared to be more frequent among subjects who had had their adapting arms hypnotically anesthetized. Subjects who had had their arms so anesthetized typically did not correct such errors while their arms were moving toward the target. However, role-playing subjects tended to correct for errors after the first few tries. Errors tended to disappear with practice. This was not the case in the hypnotic anesthesia condition, in which errors persisted throughout the exposure period.

(Received for publication August 7, 1980; revision accepted October 24, 1980.) 